\title{
LOWER BOUNDS FOR THE NUMBER OF SEMIDUALIZING COMPLEXES OVER A LOCAL RING
}

\author{
SEAN SATHER-WAGSTAFF*
}

\begin{abstract}
We investigate the set $\subseteq(R)$ of shift-isomorphism classes of semi-dualizing $R$-complexes, ordered via the reflexivity relation, where $R$ is a commutative noetherian local ring. Specifically, we study the question of whether $\subseteq(R)$ has cardinality $2^{n}$ for some $n$. We show that, if there is a chain of length $n$ in $\Im(R)$ and if the reflexivity ordering on $\Im(R)$ is transitive, then $\Im(R)$ has cardinality at least $2^{n}$, and we explicitly describe some of its order-structure. We also show that, given a local ring homomorphism $\varphi: R \rightarrow S$ of finite flat dimension, if $R$ and $S$ admit dualizing complexes and if $\varphi$ is not Gorenstein, then the cardinality of $\widetilde{S}(S)$ is at least twice the cardinality of $\widetilde{S}(R)$.
\end{abstract}

\section{Introduction}

Throughout this work $(R, \mathfrak{m})$ and $(S, \mathfrak{n})$ are commutative noetherian local rings.

A homologically finite $R$-complex $C$ is semidualizing if the natural homothety morphism $R \rightarrow \mathbf{R H o m}_{R}(C, C)$ is an isomorphism in the derived category $\mathscr{D}(R)$. (See Section 2 for background material.) Examples of semidualizing $R$-complexes include $R$ itself and a dualizing $R$-complex when one exists. The set of shift-isomorphism classes of semidualizing $R$-complexes is denoted $\subseteq(R)$, and the shift-isomorphism class of a semidualizing $R$-complex $C$ is denoted $[C]$.

Semidualizing complexes were introduced by Avramov and Foxby [2] and Christensen [4] in part to investigate the homological properties of local ring homomorphisms. Our interest in these complexes comes from their potential as tools for answering the composition question for local ring homomorphisms of finite G-dimension. Unfortunately, the utility of the semidualizing $R$-complexes is hampered by the fact that our understanding of $\subseteq(R)$ is very limited. For instance, we do not even know if the set $\subseteq(R)$ is finite; see [5] for some recent progress.

\footnotetext{
* The author was supported in part by a grant from the NSA.

Received 17 March 2009.
} 
We are interested in the following question, motivated by results from [7], wherein $|\widetilde{S}(R)|$ is the cardinality of the set $\subseteq(R)$.

Question 1.1. If $R$ is a local ring, must we have $|\subseteq(R)|=2^{n}$ for some $n \in \mathrm{N}$ ?

Each semidualizing $R$-complex $C$ gives rise to a notion of reflexivity for homologically finite $R$-complexes. For instance, each homologically finite $R$ complex of finite projective dimension is $C$-reflexive. On the other hand, a semidualizing $R$-complex $C$ is dualizing if and only if every homologically finite $R$-complex is $C$-reflexive. We order $\subseteq(R)$ using reflexivity: write $[C] \unlhd$ $[B]$ whenever $B$ is $C$-reflexive. This relation is reflexive and antisymmetric, but we do not know if it is transitive in general. A chain in $\subseteq(R)$ is a sequence $\left[C_{0}\right] \unlhd\left[C_{1}\right] \unlhd \cdots \unlhd\left[C_{n}\right]$, and such a chain has length $n$ if $\left[C_{i}\right] \neq\left[C_{j}\right]$ whenever $i \neq j$.

The main result of this paper, stated next, uses the lengths of chains in $\subseteq(R)$ to provide a lower bound of the form $2^{n}$ on the cardinality of $\subseteq(R)$. It is part of Theorem 3.3 which also contains the analogous result for the set of isomorphism classes of semidualizing $R$-modules.

THEOREM 1.2. Assume that the reflexivity ordering on $\subseteq(R)$ is transitive. If $\subseteq(R)$ admits a chain of length $n$, then $|\subseteq(R)| \geq 2^{n}$.

An alternate proof of this result is given in Corollary 4.9. One advantage of this second method is that we can describe all the reflexivity relations between these complexes in terms of combinatorial data; see Theorem 4.8.

Using the ideas from Theorem 1.2, we also prove the following comparison result which is a special case of Theorem 3.5.

Theorem 1.3. Let $\varphi: R \rightarrow S$ be a local ring homomorphism of finite flat dimension. If $R$ and $S$ admit dualizing complexes and if $\varphi$ is not Gorenstein, then $|\subseteq(S)| \geq 2|\subseteq(R)|$.

\section{Complexes and local ring homomorphisms}

This section contains definitions and background material for use in the sequel.

Definition 2.1. An $R$-complex is a sequence of $R$-module homomorphisms

$$
X=\cdots \stackrel{\partial_{n+1}^{X}}{\longrightarrow} X_{n} \stackrel{\partial_{n}^{X}}{\longrightarrow} X_{n-1} \stackrel{\partial_{n-1}^{X}}{\longrightarrow} \cdots
$$

such that $\partial_{n-1}^{X} \partial_{n}^{X}=0$ for each integer $n$. The $n$th homology module of $X$ is $\mathrm{H}_{n}(X):=\operatorname{Ker}\left(\partial_{n}^{X}\right) / \operatorname{Im}\left(\partial_{n+1}^{X}\right)$. The complex $X$ is homologically bounded when $\mathrm{H}_{n}(X)=0$ for $|n| \gg 0$. It is homologically finite if the $R$-module 
$\bigoplus_{n \in Z} \mathrm{H}_{n}(X)$ is finitely generated. We frequently identify $R$-modules with $R$ complexes concentrated in degree 0 .

Notation 2.2. We work in the derived category $\mathscr{D}(R)$. References on the subject include [9], [11], [13], [14]; see also [12]. Given two $R$-complexes $X$ and $Y$, the derived homomorphism and tensor product complexes are denoted $\mathbf{R H o m}_{R}(X, Y)$ and $X \otimes_{R}^{\mathbf{L}} Y$. Isomorphisms in $\mathscr{D}(R)$ are identified by the symbol $\simeq$, and isomorphisms up to shift are identified by $\sim$.

Definition 2.3. For each integer $n$, the $n$th Bass number of $R$ is $\mu_{R}^{n}(R)=$ $\operatorname{rank}_{R / \mathfrak{m}}\left(\operatorname{Ext}_{R}^{n}(R / \mathfrak{m}, R)\right)$, and the Bass series of $R$ is the power series $I_{R}^{R}(t)=$ $\sum_{n=0}^{\infty} \mu_{R}^{n}(R) r^{n}$.

Let $\varphi: R \rightarrow S$ be a local ring homomorphism of finite flat dimension, that is, such that $S$ admits a bounded resolution by flat $R$-modules. The Bass series of $\varphi$ is a formal Laurent series $I_{\varphi}(t)$ with nonnegative integer coefficients such that $I_{S}^{S}(t)=I_{\varphi}(t) I_{R}^{R}(t)$; see $[3,(5.1)]$ for the existence of $I_{\varphi}(t)$. The homomorphism $\varphi$ is Gorenstein at $\mathfrak{n}$ if $I_{\varphi}(t)=t^{d}$ for some integer $d$.

ExAmple 2.4. Let $\varphi: R \rightarrow S$ be a local ring homomorphism of finite flat dimension. When $\varphi$ is flat, it is Gorenstein if and only if the closed fibre $S / \mathrm{m} S$ is Gorenstein. Also, if $\varphi$ is surjective with kernel generated by an $R$-sequence, then it is Gorenstein.

Semidualizing complexes, defined next, are our main objects of study.

Definition 2.5. A homologically finite $R$-complex $C$ is semidualizing if the natural homothety morphism $\chi_{C}^{R}: R \rightarrow \mathbf{R H o m}_{R}(C, C)$ is an isomorphism in $\mathscr{D}(R)$. An $R$-complex $D$ is dualizing if it is semidualizing and has finite injective dimension. Let $\subseteq(R)$ denote the set of shift-isomorphism classes of semidualizing $R$-complexes, and let $[C]$ denote the shift-isomorphism class of a semidualizing $R$-complex $C$.

When $C$ is a finitely generated $R$-module, it is semidualizing if and only if $\operatorname{Ext}_{R}^{\geq 1}(C, C)=0$ and the natural homothety map $R \rightarrow \operatorname{hom}_{R}(C, C)$ is an isomorphism. Let $\widetilde{S}_{0}(R)$ denote the set of isomorphism classes of semidualizing $R$-modules, and let $[C]$ denote the isomorphism class of a semidualizing $R$-module $C$. The natural identification of an $R$-module with an $R$-complex concentrated in degree 0 provides a natural inclusion $\Im_{0}(R) \subseteq \subseteq(R)$.

REMARK 2.6. Let $\varphi: R \rightarrow S$ be a local ring homomorphism of finite flat dimension, and fix semidualizing $R$-complexes $B, C$. The complex $S \otimes_{R}^{\mathrm{L}} C$ is semidualizing for $S$ by [4, (5.7)]. The complex $S \otimes_{R}^{\mathbf{L}} C$ is dualizing for $S$ if and only if $C$ is dualizing for $R$ and $\varphi$ is Gorenstein by [1, (5.1)]. We have $S \otimes_{R}^{\mathbf{L}} B \simeq S \otimes_{R}^{\mathbf{L}} C$ in $\mathscr{D}(S)$ if and only if $B \simeq C$ in $\mathscr{D}(R)$ by [6, (1.10)]. Hence, 
the function $\subseteq(\varphi): \subseteq(R) \rightarrow \subseteq(S)$ given by $[C] \mapsto\left[S \otimes_{R}^{\mathrm{L}} C\right]$ is well-defined and injective.

The next definition is due to Christensen [4] and Hartshorne [11] and will be used primarily to compare semidualizing complexes.

Definition 2.7. Let $C$ be a semidualizing $R$-complex. A homologically finite $R$-complex $X$ is $C$-reflexive when the $R$-complex $\operatorname{RHom}_{R}(X, C)$ is homologically finite, and the natural biduality morphism $\delta_{X}^{C}: X \rightarrow$ $\mathbf{R} \operatorname{Hom}_{R}\left(\mathbf{R H o m}_{R}(X, C), C\right)$ is an isomorphism in $\mathscr{D}(R)$. Define an order on $\Im(R)$ by writing $[C] \unlhd[B]$ when $B$ is $C$-reflexive. Also, write $[C] \triangleleft[B]$ when $[C] \unlhd[B]$ and $[C] \neq[B]$. For each $[C] \in \varsigma_{(R)}$ set $\widetilde{S}_{C}(R)=\{[B] \in \widetilde{S}(R) \mid$ $[C] \unlhd[B]\}$.

Remark 2.8. Let $A, B$ and $C$ be semidualizing $R$-complexes.

1. If $B$ is $C$-reflexive, then $\operatorname{RHom}_{R}(B, C)$ is semidualizing and $C$-reflexive by $[4,(2.12)]$. Thus, the map $\Phi_{C}: \Im_{C}(R) \rightarrow \widetilde{\subseteq}_{C}(R)$ given by $[B] \mapsto$ $\left[\mathbf{R} \operatorname{Hom}_{R}(B, C)\right]$ is well-defined. By definition, this map is also an involution (i.e., $\Phi_{C}^{2}=\operatorname{id}_{\Phi_{C}(R)}$ ) and hence it is bijective. From [6, (3.9)] we know that $\Phi_{C}$ is reverses the reflexivity ordering: if $[A],[B] \in \widetilde{S}_{C}(R)$, then $[A] \unlhd[B]$ if and only if $\Phi_{C}([B]) \unlhd \Phi_{C}([A])$, that is, if and only if $\left[\mathbf{R} \operatorname{Hom}_{R}(B, C)\right] \unlhd\left[\mathbf{R} \operatorname{Hom}_{R}(A, C)\right]$.

2. Assume that $C$ is a semidualizing $R$-module. Using [6, (3.5)] we see that, if $B$ is $C$-reflexive, then $B$ is isomorphic up to shift with a semidualizing $R$-module, and hence so is $\mathbf{R H o m}_{R}(B, C)$. In particular, we have $\subseteq_{C}(R) \subseteq$ $\widetilde{S}_{0}(R)$.

3. If $D$ is a dualizing $R$-complex, then $[D] \unlhd[C]$ by [11, (V.2.1)], i.e., we have $\widetilde{\subseteq}_{D}(R)=\subseteq(R)$.

4. Let $X$ be an $R$-complex such that $\mathrm{H}_{i}(X)$ is finitely generated for each $i$ and $\mathrm{H}_{i}(X)=0$ for $i \ll 0$. If $C \otimes_{R}^{\mathbf{L}} C \otimes_{R}^{\mathbf{L}} X$ is semidualizing, then $C \sim R$ by $[7,(3.2)]$.

5. By $[10,(3.3)]$, given a chain $\left[C_{n}\right] \triangleleft\left[C_{n-1}\right] \triangleleft \cdots \triangleleft\left[C_{0}\right]$ in $\subseteq(R)$, one has

$$
C_{n} \simeq C_{0} \otimes_{R}^{\mathbf{L}} \mathbf{R H o m}_{R}\left(C_{0}, C_{1}\right) \otimes_{R}^{\mathbf{L}} \cdots \otimes_{R}^{\mathbf{L}} \mathbf{R} \operatorname{Hom}_{R}\left(C_{n-1}, C_{n}\right) .
$$

REMARK 2.9. Let $\varphi: R \rightarrow S$ be a local ring homomorphism of finite flat dimension. The map $\subseteq(\varphi): \subseteq(R) \rightarrow \subseteq(S)$ from Remark 2.6 respects the reflexivity orderings perfectly by $[6,(4.8)]$ : if $[B],[C] \in \Im_{(}(R)$, then $[C] \unlhd$ $[B]$ if and only if $\subseteq(\varphi)([C]) \unlhd \subseteq(\varphi)([B])$ that is, if and only if $\left[S \otimes_{R}^{\mathbf{L}} C\right] \unlhd$ $\left[S \otimes_{R}^{\mathbf{L}} B\right]$.

The next definition is due to Avramov and Foxby [2] and Christensen [4]. 
Definition 2.10. Let $C$ be a semidualizing $R$-complex. A homologically boun-ded $R$-complex $X$ is in the Bass class with respect to $C$, denoted $\mathscr{B}_{C}(R)$, when the $R$-complex $\mathbf{R H o m}_{R}(C, X)$ is homologically bounded and the natural evaluation morphism $\xi_{X}^{C}: C \otimes_{R}^{\mathbf{L}} \mathbf{R} \operatorname{Hom}_{R}(C, X) \rightarrow X$ is an isomorphism in $\mathscr{D}(R)$. A homologically bounded $R$-complex $X$ is in the Auslander class with respect to $C$, denoted $\mathscr{A}_{C}(R)$, when the $R$-complex $C \otimes_{R}^{\mathrm{L}} X$ is homologically bounded and the natural morphism $\gamma_{X}^{C}: X \rightarrow \operatorname{RHom}_{R}\left(C, C \otimes_{R}^{\mathbf{L}} X\right)$ is an isomorphism in $\mathscr{D}(R)$.

Remark 2.11. Let $B$ and $C$ be semidualizing $R$-complexes. Then $B \in$ $\mathscr{B}_{C}(R)$ if and only if $[B] \unlhd[C]$; see [8, (1.3)]. One has $B \in \mathscr{A}_{C}(R)$ if and only if $B \otimes_{R}^{\mathbf{L}} C$ is a semidualizing $R$-complex by [8, (4.8)].

\section{Bounding the number of elements in $\subseteq(R)$}

This section contains the proofs of Theorems 1.2 and 1.3 from the introduction. We begin with two lemmas.

Lemma 3.1. Let $A, B$ and $C$ be semidualizing $R$-complexes such that $B$ and $C$ are $A$-reflexive and $B$ is $C$-reflexive. If $C \not A$, then $\operatorname{RHom}_{R}(B, A)$ is not $C$-reflexive.

Proof. Remark 2.8.1 implies that $\mathbf{R H o m}_{R}(B, A)$ and $\mathbf{R} \operatorname{Hom}_{R}(C, A)$ are semidualizing $R$-complexes and that $\mathbf{R H o m}_{R}(C, A)$ is $\mathbf{R H o m}_{R}(B, A)$-reflexive. In the next sequence, the first isomorphism is from Remark 2.8.5:

$$
\begin{aligned}
\mathbf{R} H_{i} & (B, A) \\
& \simeq \mathbf{R H o m}_{R}(C, A) \otimes_{R}^{\mathbf{L}} \mathbf{R H o m}_{R}\left(\mathbf{R H o m}_{R}(C, A), \mathbf{R H o m}_{R}(B, A)\right) \\
& \simeq \mathbf{R H o m}_{R}(C, A) \otimes_{R}^{\mathbf{L}} \mathbf{R H o m}_{R}\left(\mathbf{R H o m}_{R}(C, A) \otimes_{R}^{\mathbf{L}} B, A\right) \\
& \simeq \mathbf{R H o m}_{R}(C, A) \otimes_{R}^{\mathbf{L}} \mathbf{R H o m}_{R}\left(B, \mathbf{R H o m}_{R}\left(\mathbf{R H o m}_{R}(C, A), A\right)\right) \\
& \simeq \mathbf{R H o m}_{R}(C, A) \otimes_{R}^{\mathbf{L}} \mathbf{R H o m}_{R}(B, C) .
\end{aligned}
$$

The second and third isomorphisms are Hom-tensor adjointness, and the fourth isomorphism comes from the fact that $C$ is $A$-reflexive.

Set $X=\mathbf{R H o m}_{R}(B, C) \otimes_{R}^{\mathbf{L}} \mathbf{R} \operatorname{Hom}_{R}\left(\mathbf{R H o m}_{R}(B, A), C\right)$, and suppose that the complex $\operatorname{RHom}_{R}(B, A)$ is $C$-reflexive. Remark 2.8.5 explains the first isomorphism in the next sequence, and the second isomorphism is from the previous display

$$
\begin{aligned}
C & \simeq \mathbf{R H o m}_{R}(B, A) \otimes_{R}^{\mathbf{L}} \mathbf{R H o m}_{R}\left(\mathbf{R H o m}_{R}(B, A), C\right) \\
& \simeq \mathbf{R H o m}_{R}(C, A) \otimes_{R}^{\mathbf{L}} \mathbf{R H o m}_{R}(B, C) \otimes_{R}^{\mathbf{L}} \mathbf{R H o m}_{R}\left(\mathbf{R H o m}_{R}(B, A), C\right) \\
& \simeq \mathbf{R H o m}_{R}(C, A) \otimes_{R}^{\mathbf{L}} X .
\end{aligned}
$$


Similarly, this yields the next sequence

$$
\begin{aligned}
A & \simeq \mathbf{R H o m}_{R}(C, A) \otimes_{R}^{\mathbf{L}} C \\
& \simeq \mathbf{R H o m}_{R}(C, A) \otimes_{R}^{\mathbf{L}} \mathbf{R} \operatorname{Hom}_{R}(C, A) \otimes_{R}^{\mathbf{L}} X .
\end{aligned}
$$

It follows from Remark 2.8.4 that $\operatorname{RHom}_{R}(C, A) \sim R$ and hence

$$
C \simeq \operatorname{RHom}_{R}\left(\operatorname{RHom}_{R}(C, A), A\right) \sim \operatorname{RHom}_{R}(R, A) \simeq A
$$

since $C$ is $A$-reflexive. This contradicts the assumption $C \nsim A$.

Note that the hypothesis $\subseteq_{C}(R) \subseteq \widetilde{S}_{A}(R)$ from the next result is satisfied when either $A$ is dualizing for $R$ or the reflexivity ordering on $\subseteq(R)$ is transitive.

Lemma 3.2. Let $A$ and $C$ be semidualizing $R$-complexes such that $C$ is $A$ reflexive and $C \neq A$. Assume that $\Im_{C}(R) \subseteq \Im_{A}(R)$, e.g., that the reflexivity ordering on $\subseteq(R)$ is transitive. The injection $\Phi_{A}: \Im_{A}(R) \rightarrow \Xi_{A}(R)$ given by $[B] \mapsto\left[\mathbf{R H o m}_{R}(B, A)\right]$ maps $\widetilde{\subseteq}_{C}(R)$ into $\widetilde{\Xi}_{A}(R) \backslash \widetilde{\Xi}_{C}(R)$. In particular $\left|\Im_{A}(R)\right| \geq 2\left|\Im_{C}(R)\right|$.

Proof. The first conclusion is a reformulation of Lemma 3.1; see also Remark 2.8.1. For the second conclusion, note that $\Phi_{A}$ is injective by Remark 2.8.1, so $\left|\Phi_{A}\left(\widetilde{\Xi}_{C}(R)\right)=\right| \widetilde{\Xi}_{C}(R) \mid$. Since $\Phi_{A}\left(\widetilde{\Xi}_{C}(R)\right) \subset \widetilde{\Xi}_{A}(R)-$ $\widetilde{\Xi}_{C}(R)$, we conclude that $\widetilde{\Xi}_{C}(R)$ and $\Phi_{A}\left(\widetilde{S}_{C}(R)\right)$ are disjoint subsets of $\widetilde{S}_{A}(R)$ such that $\left|\widetilde{S}_{C}(R)\right|=\left|\Phi_{A}\left(\widetilde{S}_{C}(R)\right)\right|$. The second conclusion now follows.

The next result contains Theorem 1.2 from the introduction.

THEOREM 3.3. Assume that $\subseteq(R)$ admits a chain $\left[C_{n}\right] \triangleleft\left[C_{n-1}\right] \triangleleft \cdots \triangleleft\left[C_{0}\right]$ such that $\widetilde{S}_{C_{0}}(R) \subseteq \cdots \subseteq \widetilde{S}_{C_{n-1}}(R) \subseteq \widetilde{S}_{C_{n}}(R)$. Then $\left|\subseteq_{(}(R)\right| \geq 2^{n}$. If $\left[C_{n}\right] \in \widetilde{S}_{0}(R)$, then $\left|\Im_{0}(R)\right| \geq 2^{n}$.

Proof. For the first statement, we show by induction on $j$ that $\left|\Im_{C_{j}}(R)\right| \geq$ $2^{j}$. For $j=0$ this is straightforward. For the inductive step assume that $\left|\widetilde{S}_{C_{j}}(R)\right| \geq 2^{j}$. Lemma 3.2 implies that $\left|\widetilde{S}_{C_{j+1}}(R)\right| \geq 2\left|\widetilde{\subseteq}_{C_{j}}(R)\right| \geq 2^{j+1}$ as desired.

When $\left[C_{n}\right] \in \Im_{0}(R)$, we have $\Im_{C_{j}}(R) \subseteq \Im_{C_{n}}(R) \subseteq \Im_{0}(R)$ for $j=$ $0, \ldots, n$ by Remark 2.8.2. Thus, the second statement is proved like the first statement.

We next provide an explicit description of the $2^{n}$ semidualizing complexes that are guaranteed to exist by Theorem 3.3. A second description (in the case $C_{0} \simeq R$ ) is given in Theorem 4.8. 
Remark 3.4. Assume that $\subseteq(R)$ admits a chain $\left[C_{n}\right] \triangleleft\left[C_{n-1}\right] \triangleleft \cdots \triangleleft\left[C_{0}\right]$ such that $\widetilde{S}_{C_{0}}(R) \subseteq \cdots \subseteq \Im_{C_{n-1}}(R) \subseteq \Im_{C_{n}}(R)$. Given $R$-complexes $C$ and $B$, set $C^{\dagger_{B}}=\mathbf{R H o m}_{R}(C, B)$.

The next diagram shows the steps $n=0,1,2,3$ of the induction argument in the proof of Theorem 3.3, with some of the reflexivity relations indicated with edges:
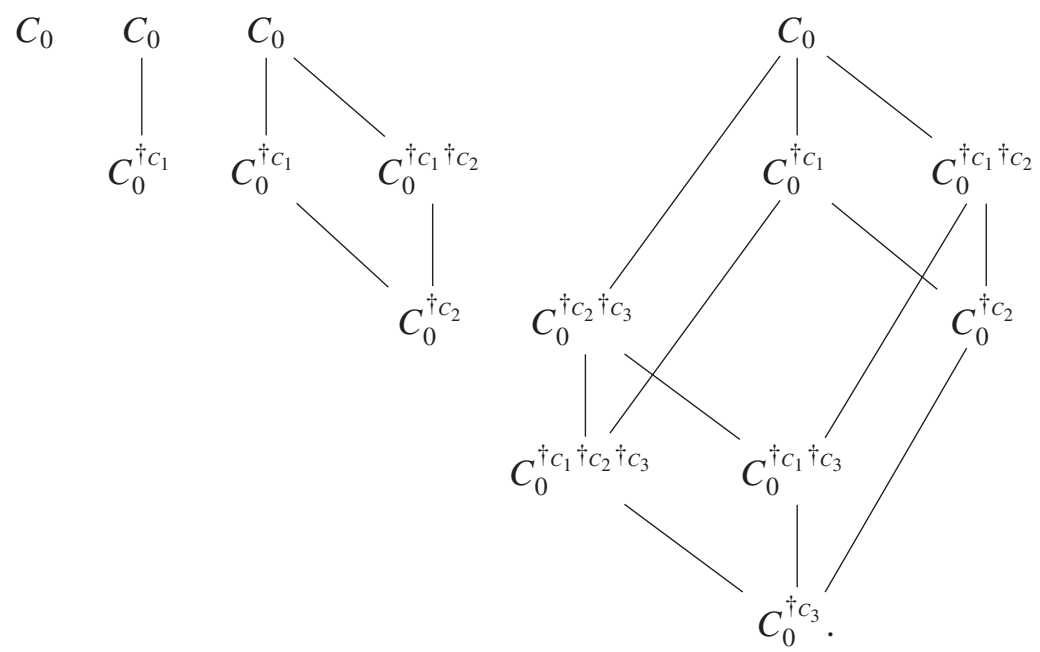

More generally, for each sequence of integers $\underline{i}=\left\{i_{1}, \ldots, i_{j}\right\}$ such that $j \geq 0$ and $1 \leq i_{1}<\cdots<i_{j} \leq n$, the $R$-complex $C_{\underline{i}}=C_{0}^{\dagger c_{i_{1}}{ }^{\dagger} c_{i_{2}}{ }^{\dagger} c_{i_{3}} \cdots \dagger c_{i_{j}}}$ is semidualizing. (When $j=0$ we have $C_{\underline{i}}=C_{\emptyset}=\bar{C}_{0}$.) The classes $\left[C_{\underline{i}}\right]$ are parametrized by the allowable sequences $\underline{i}$, of which there are exactly $2^{\vec{n}}$. These are precisely the $2^{n}$ classes constructed in the proof of Theorem 3.3.

If the $C_{i}$ are all modules, then we may replace $\mathbf{R H o m}$ with Hom to obtain a description of the semidualizing modules that the theorem guarantees to exist.

The next result contains Theorem 1.3 from the introduction.

Theorem 3.5. Let $\varphi: R \rightarrow S$ be a local ring homomorphism of finite flat dimension. Assume that $\subseteq(R)$ has a unique minimal element $[A]$. (For instance, this holds when $R$ admits a dualizing complex.) If $S$ admits a dualizing complex $D^{S}$ and if $\varphi$ is not Gorenstein at $\mathfrak{n}$, then $|\subseteq(S)| \geq 2|\subseteq(R)|$.

Proof. Let $\subseteq(\varphi): \subseteq(R) \rightarrow \subseteq(S)$ be the induced map from Remark 2.6. Our assumption on $A$ implies that $\subseteq(R)=\Xi_{A}(R)$. Remark 2.9 provides the first containment in the next sequence while Remark 2.8.3 explains the last equality

$$
\widetilde{S}(\varphi)(\widetilde{S}(R))=\widetilde{S}(\varphi)\left(\widetilde{S}_{A}(R)\right) \subseteq \widetilde{S}_{S \otimes_{R}^{\mathrm{L} A}}(S) \subseteq \widetilde{S}(S)=\widetilde{S}_{D^{S}}(S) .
$$


Since $\varphi$ is not Gorenstein, Remark 2.6 implies $D^{S} \nsucc S \otimes_{R}^{\mathbf{L}} A$. The injectivity of $\subseteq(\varphi)$ explains the first inequality in the next sequence

$$
2|\Im(R)|=2\left|\Im_{A}(R)\right| \leq 2\left|\Im_{S \otimes_{R}^{\mathrm{L}} A}(S)\right| \leq\left|\Im_{D^{S}}(S)\right|=|\Im(S)|
$$

while the second inequality is from Lemma 3.2.

\section{Structure on the Set of Semidualizing $R$-complexes}

This section is devoted to providing a second description of the $2^{n}$ semidualizing complexes that are guaranteed to exist by Theorem 3.3. This description is contained in Theorems 4.7 and 4.8. It says, in particular, that these complexes form a dual version of the "LCM lattice" on $n$ formal letters. Note that the version for semidualizing modules has the same form with derived functors replaced by non-derived functors; hence, we do not state it explicitly. We begin with some notation for use throughout the section and five supporting lemmas.

Assumption 4.1. Assume throughout this section that $\widetilde{S}(R)$ admits a chain $\left[C_{n}\right] \triangleleft\left[C_{n-1}\right] \triangleleft \cdots \triangleleft\left[C_{0}\right]$. For $i=1, \ldots, n$ set $B_{i}=\mathbf{R} \operatorname{Hom}_{R}\left(C_{i-1}, C_{i}\right)$. Set $B_{\emptyset}=C_{0}$. For each sequence $\underline{i}=\left\{i_{1}, \ldots, i_{j}\right\}$ such that $1 \leq i_{1}<\cdots<i_{j} \leq n$ and $j \geq 1$, set $B_{\underline{i}}=B_{i_{1}} \otimes_{R}^{\mathrm{L}} \cdots \otimes_{R}^{\mathrm{L}} B_{i_{j}}$. (When $j=1$, we have $B_{\underline{i}}=B_{\left\{i_{1}\right\}}=$ $B_{i_{1}}$.)

Lemma 4.2. Under the hypotheses of Assumption 4.1, if $1 \leq i<j \leq n$, then

$$
\mathbf{R H o m}_{R}\left(C_{i-1}, C_{j-1}\right) \otimes_{R}^{\mathbf{L}} B_{j} \simeq \mathbf{R H o m}_{R}\left(C_{i-1}, C_{j}\right) .
$$

Proof. Consider the following sequence of isomorphisms:

$$
\begin{aligned}
\mathbf{R H o m}_{R}\left(B_{j}, \mathbf{R H o m}_{R}\left(C_{i-1}, C_{j}\right)\right) \\
\quad \simeq \mathbf{R H o m}_{R}\left(\mathbf{R H o m}_{R}\left(C_{j-1}, C_{j}\right), \mathbf{R} \operatorname{Rom}_{R}\left(C_{i-1}, C_{j}\right)\right) \\
\quad \simeq \mathbf{R H o m}_{R}\left(\mathbf{R H o m}_{R}\left(C_{j-1}, C_{j}\right) \otimes_{R}^{\mathbf{L}} C_{i-1}, C_{j}\right) \\
\quad \simeq \mathbf{R H o m}_{R}\left(C_{i-1}, \mathbf{R} \operatorname{Hom}_{R}\left(\mathbf{R} \operatorname{Hom}_{R}\left(C_{j-1}, C_{j}\right), C_{j}\right)\right) \\
\quad \simeq \mathbf{R H o m}_{R}\left(C_{i-1}, C_{j-1}\right) .
\end{aligned}
$$

The first two isomorphisms are from Hom-tensor adjointness and the commutativity of tensor product. The third isomorphism is from the condition $\left[C_{j}\right] \unlhd\left[C_{j-1}\right]$. Since the $R$-complexes $B_{j}, \mathbf{R H o m}_{R}\left(C_{i-1}, C_{j}\right)$ and $\mathbf{R H o m}_{R}\left(C_{i-1}, C_{j-1}\right)$ are semidualizing, it follows from [8, (1.3)] that $\left[\mathbf{R} \operatorname{Hom}_{R}\left(C_{i-1}, C_{j}\right)\right] \unlhd\left[B_{j}\right]$ so the first isomorphism in the next sequence is from Remark 2.8.5:

$$
\begin{aligned}
\mathbf{R H o m}_{R}\left(C_{i-1}, C_{j}\right) & \simeq B_{j} \otimes_{R}^{\mathbf{L}} \mathbf{R H o m}_{R}\left(B_{j}, \mathbf{R H o m}_{R}\left(C_{i-1}, C_{j}\right)\right) \\
& \simeq B_{j} \otimes_{R}^{\mathbf{L}} \mathbf{R H o m}_{R}\left(C_{i-1}, C_{j-1}\right)
\end{aligned}
$$


The second isomorphism is from the first displayed sequence in this proof.

Lemma 4.3. Under the hypotheses of Assumption 4.1, if $1 \leq i<i+p \leq n$, then

$$
B_{\{i, i+1, \ldots, i+p\}} \simeq \mathbf{R H o m}_{R}\left(C_{i-1}, C_{i+p}\right) .
$$

Proof. We proceed by induction on $p$. The base case is when $p=1$. Setting $j=i+1$ in Lemma 4.2 yields the first isomorphism in the next sequence

$\mathbf{R H o m}_{R}\left(C_{i-1}, C_{i+1}\right) \simeq B_{i+1} \otimes_{R}^{\mathbf{L}} \mathbf{R} \operatorname{Hom}_{R}\left(C_{i-1}, C_{i}\right) \simeq B_{i+1} \otimes_{R}^{\mathbf{L}} B_{i} \simeq B_{\{i, i+1\}}$.

The remaining isormorphisms are by definition.

For the inductive step, assume that $B_{\{i, i+1, \ldots, i+p-1\}} \simeq \mathbf{R} \operatorname{Hom}_{R}\left(C_{i-1}, C_{i+p-1}\right)$. This assumption explains the second isomorphism in the next sequence

$$
\begin{aligned}
B_{\{i, i+1, \ldots, i+p\}} & \simeq B_{i} \otimes_{R}^{\mathbf{L}} \cdots \otimes_{R}^{\mathbf{L}} B_{i+p-1} \otimes_{R}^{\mathbf{L}} B_{i+p} \\
& \simeq \mathbf{R H o m}_{R}\left(C_{i-1}, C_{i+p-1}\right) \otimes_{R}^{\mathbf{L}} B_{i+p} \\
& \simeq \mathbf{R H o m}_{R}\left(C_{i-1}, C_{i+p}\right) .
\end{aligned}
$$

The first and third isomorphisms are by definition, and the fourth isomorphism is from Lemma 4.2.

Lemma 4.4. Assume that $\Im_{C_{i}}(R) \subseteq \Im_{C_{j-1}}(R)$. Under the hypotheses of Assumption 4.1, if $1 \leq i<j-1 \leq n-1$, then

$$
\left.B_{\{i, j\}} \simeq \mathbf{R H o m}_{R}\left(\operatorname{RHom}_{R}\left(B_{i}, C_{j-1}\right), C_{j}\right)\right) .
$$

(In the notation from Remark 3.4, this reads $B_{\{i, j\}} \simeq B_{i}^{\dagger c_{j-1}^{\dagger} c_{j}} \simeq C_{i-1}^{\dagger C_{i} \dagger c_{j-1} \dagger c_{j}}$.)

Proof. The proof is similar to that for Lemma 4.2. The main point is the following sequence of isomorphisms wherein the last two isomorphisms are from Hom-tensor adjointness and the commutativity of tensor product:

$$
\begin{aligned}
B_{i} & \simeq \mathbf{R H o m}_{R}\left(\mathbf{R H o m}_{R}\left(B_{i}, C_{j-1}\right), C_{j-1}\right) \\
& \simeq \mathbf{R H o m}_{R}\left(\mathbf{R H o m}_{R}\left(B_{i}, C_{j-1}\right), \mathbf{R H o m}_{R}\left(B_{j}, C_{j}\right)\right) \\
& \simeq \mathbf{R H o m}_{R}\left(B_{j} \otimes_{R}^{\mathbf{L}} \mathbf{R} \operatorname{Rom}_{R}\left(B_{i}, C_{j-1}\right), C_{j}\right) \\
& \simeq \mathbf{R H o m}_{R}\left(B_{j}, \mathbf{R H o m}_{R}\left(\mathbf{R H o m}_{R}\left(B_{i}, C_{j-1}\right), C_{j}\right)\right) .
\end{aligned}
$$

The first isomorphism is from the chain $\left[C_{j-1}\right] \unlhd\left[C_{i}\right] \unlhd\left[\mathbf{R} \operatorname{Hom}_{R}\left(C_{i-1}, C_{i}\right)\right]$ $=\left[B_{i}\right]$, using the containment $\widetilde{S}_{C_{i}}(R) \subseteq \widetilde{S}_{C_{j-1}}(R)$; see Remark 2.8.1. The second isomorphism is from the condition $\left[C_{j}\right] \unlhd\left[C_{j-1}\right]$ and the definition of $B_{j}$. 
Lemma 4.5. Assume that $\Im_{C_{1}}(R) \subseteq \cdots \subseteq \subseteq_{C_{n-1}}(R) \subseteq \Im_{C_{n}}(R)$. Under the hypotheses of Assumption 4.1, each of the $R$-complexes $B_{i}$ is semidualizing.

Proof. We proceed by induction on $n$, the length of the chain in $\subseteq(R)$. The base cases $n=0,1$ are routine. Indeed, when $n=0$, we have only one $B_{\underline{i}}$, namely $B_{\emptyset}=C_{0}$. When $n=1$, we have only two $B_{\underline{i}}$, namely $B_{\{1\}}=B_{1}$ and $B_{\emptyset}=C_{0}$.

Now, assume that $n \geq 2$ and that the result holds for chains of length $n-1$. Also, assume without loss of generality that $i \neq \emptyset$. We have two cases.

Case 1: If $i_{2}=i_{1}+1$, then the first isomorphism in the next sequence is by definition, and the second isomorphism is from Lemma 4.3:

$$
\begin{aligned}
B_{i} & \simeq B_{i_{1}} \otimes_{R}^{\mathbf{L}} B_{i_{1}+1} \otimes_{R}^{\mathbf{L}} B_{i_{3}} \otimes_{R}^{\mathbf{L}} \cdots \otimes_{R}^{\mathbf{L}} B_{i_{j}} \\
& \simeq \mathbf{R} \operatorname{Hom}_{R}\left(C_{i_{1}-1}, C_{i_{1}+1}\right) \otimes_{R}^{\mathbf{L}} B_{i_{3}} \otimes_{R}^{\mathbf{L}} \cdots \otimes_{R}^{\mathbf{L}} B_{i_{j}}
\end{aligned}
$$

Applying our induction hypothesis to the chain $\left[C_{n}\right] \triangleleft \cdots \triangleleft\left[C_{i_{1}+1}\right] \triangleleft\left[C_{i_{1}-1}\right] \triangleleft$ $\cdots \triangleleft\left[C_{0}\right]$, we conclude that the tensor product in the final line of this sequence is semidualizing. Hence $B_{i}$ is semidualizing in this case.

Case 2: If $i_{2}>i_{1}+1$, then the first isomorphism in the next sequence is by definition, and the second isomorphism is from Lemma 4.4:

$B_{i}$

$\simeq B_{i_{1}} \otimes_{R}^{\mathbf{L}} B_{i_{2}} \otimes_{R}^{\mathbf{L}} B_{i_{3}} \otimes_{R}^{\mathbf{L}} \cdots \otimes_{R}^{\mathbf{L}} B_{i_{j}}$

$\simeq \mathbf{R H o m}_{R}\left(\mathbf{R H o m}_{R}\left(\mathbf{R H o m}_{R}\left(C_{i_{1}-1}, C_{i_{1}}\right), C_{i_{2}-1}\right), C_{i_{2}}\right) \otimes_{R}^{\mathbf{L}} B_{i_{3}} \otimes_{R}^{\mathbf{L}} \cdots \otimes_{R}^{\mathbf{L}} B_{i_{j}}$

Applying our induction hypothesis to the chain

$$
\left[C_{n}\right] \triangleleft \cdots \triangleleft\left[C_{i_{2}}\right] \triangleleft\left[\mathbf{R} \operatorname{Hom}_{R}\left(\mathbf{R} \operatorname{Hom}_{R}\left(C_{i_{1}-1}, C_{i_{1}}\right), C_{i_{2}-1}\right)\right]
$$

we conclude again that $B_{\underline{i}}$ is semidualizing in this case.

Lemma 4.6. Assume that $\widetilde{\subseteq}_{C_{1}}(R) \subseteq \cdots \subseteq \widetilde{\subseteq}_{C_{n-1}}(R) \subseteq \widetilde{\subseteq}_{C_{n}}(R)$. Under the hypotheses of Assumption 4.1, if $\underline{\underline{s}}=\left\{s_{1}, \ldots, s_{1}\right\}$ is a sequence of integers such that $1 \leq s_{1}<\cdots<s_{t} \leq n$ and $\underline{i} \supseteq \underline{s}$, then $\left[B_{\underline{i}}\right] \unlhd\left[B_{\underline{s}}\right]$ and $\operatorname{RHom}_{R}\left(B_{\underline{s}}, B_{\underline{i}}\right) \simeq B_{\underline{i}-\underline{s}}$.

Proof. By Lemma 4.5 , the $R$-complexes $B_{\underline{i}}, B_{\underline{s}}$ and $B_{\underline{i} \backslash \underline{s}}$ are semidualizing. By definition, we have $B_{\underline{s}} \simeq B_{\underline{i}} \otimes_{R}^{\mathrm{L}} B_{\underline{i} \backslash \underline{s}}$, so the result follows from [10, (3.5)].

The following theorem compares the complexes constructed in this section to those from the previous section.

Theorem 4.7. Assume that $\widetilde{S}_{C_{1}}(R) \subseteq \cdots \subseteq \subseteq_{C_{n-1}}(R) \subseteq \subseteq_{C_{n}}(R)$. Under the hypotheses of Assumption 4.1, if $C_{0} \simeq R$, then the R-complexes $B_{i}$ are precisely the $2^{n}$ complexes constructed in Theorem 3.3. 
Proof. We proceed by induction on $n$, the length of the chain in $\subseteq(R)$. The base case $n=0$ is straightforward: The only $B_{\underline{i}}$ is $B_{\emptyset}=C_{0} \simeq R$, which is the only module constructed in the proof of Theorem 3.3.

For the inductive step, assume that $n \geq 1$ and that the result holds for the chain $\left[C_{n-1}\right] \triangleleft \cdots \triangleleft\left[C_{0}\right]$. Then the $R$-complexes $B_{i}$ with $i_{j} \leq n-1$ are precisely the $2^{n-1}$ complexes constructed in Theorem 3.3 for this chain.

The remaining $2^{n-1}$ semidualizing $R$-complexes constructed in Theorem 3.3 (according to the proof) for the chain $\left[C_{n}\right] \triangleleft\left[C_{n-1}\right] \triangleleft \cdots \triangleleft\left[C_{0}\right]$ are then of the form $\mathbf{R H o m}_{R}\left(B_{\underline{i}}, C_{n}\right)$. Thus, it remains to show that each of these complexes is of the form $B_{\underline{s}}$. This is shown in the following sequence wherein we use the notation $[n]=\{1,2, \ldots, n\}$ :

$$
\begin{aligned}
\mathbf{R H o m}_{R}\left(B_{\underline{i}}, C_{n}\right) & \simeq \mathbf{R H o m}_{R}\left(B_{\underline{i}}, B_{1} \otimes_{R}^{\mathbf{L}} \cdots \otimes_{R}^{\mathbf{L}} B_{n}\right) \\
& \simeq \mathbf{R H o m}_{R}\left(B_{\underline{i}}, B_{[n]}\right) \simeq B_{[n] \backslash \underline{i}}
\end{aligned}
$$

The first isomorphism follows from Remark 2.8.5 using the assumption $C_{0} \simeq$ $R$. The second isomorphism is by definition. The third isomorphism is from Lemma 4.6.

The next result provides a purely combinatorial description of the reflexivity ordering on the set of $\left[B_{\underline{i}}\right]$.

Theorem 4.8. Assume that $\widetilde{\subseteq}_{C_{1}}(R) \subseteq \cdots \subseteq \subseteq_{C_{n-1}}(R) \subseteq \subseteq_{C_{n}}(R)$. Under the hypotheses of Assumption 4.1, the following conditions are equivalent:

(i) One has $B_{\underline{i}} \unlhd B_{\underline{s}}$;

(ii) One has $B_{\underline{s}} \in \mathscr{B}_{B_{\underline{i}}}(R)$; and

(iii) One has $\underline{i} \supseteq \underline{s}$.

Proof. The equivalence (i) $\Longleftrightarrow$ (ii) is from Remark 2.11; and the implication (iii) $\Longrightarrow$ (i) is contained in Lemma 4.6.

(i) $\Longrightarrow$ (iii): Assume that $\left[B_{\underline{i}}\right] \unlhd\left[B_{\underline{s}}\right]$. Lemma 4.6 implies that $\left[B_{\underline{s} \cup \underline{i}}\right] \unlhd$ $\left[B_{\underline{s}}\right]$ and $\left[B_{\underline{i}}\right] \unlhd\left[B_{\underline{i} \backslash \underline{s}}\right]$, and furthermore that $\operatorname{RHom}_{R}\left(B_{\underline{s}}, B_{\underline{s}} \cup \underline{\underline{i}}\right) \simeq \bar{B}_{\underline{i} \backslash \underline{\underline{s}}}$. Since the reflexivity ordering on $\subseteq(R)$ is transitive, we have

$$
\left[B_{\underline{s} \cup \underline{i}}\right] \unlhd\left[B_{\underline{s}}\right] \unlhd\left[\mathbf{R H o m}_{R}\left(B_{\underline{s}}, B_{\underline{s}} \cup \underline{i}\right)\right] .
$$

From $[7,(3.3 . a)]$ we conclude that $\left[B_{\underline{s} \cup \underline{i}}\right]=\left[B_{\underline{s}}\right]$, and hence the first equality in the next sequence:

$$
[R]=\left[\operatorname{RHom}_{R}\left(B_{\underline{s}}, B_{\underline{s} \cup \underline{i}}\right)\right]=\left[B_{\underline{i} \backslash \underline{s}}\right] .
$$

It is straightforward to show that this implies that $\underline{i} \backslash \underline{s}=\emptyset$, and thus $\underline{i} \subseteq \underline{s}$. 
COROLlaRY 4.9. Assume that $\Xi_{C_{1}}(R) \subseteq \cdots \subseteq \Im_{C_{n-1}}(R) \subseteq \Im_{C_{n}}(R)$. Under the hypotheses of Assumption 4.1, one has $\left[B_{\underline{i}}\right]=\left[B_{\underline{s}}\right]$ if and only if $\underline{i}=\underline{s}$. In particular, there are exactly $2^{n}$ classes of the form $\left[\bar{B}_{\underline{i}}\right]$.

Proof. One implication of the biconditional statement is straightforward. For the converse, assume that $\left[B_{\underline{i}}\right]=\left[B_{\underline{s}}\right]$. Then $\left[B_{\underline{i}}\right] \unlhd\left[B_{\underline{s}}\right] \unlhd\left[B_{\underline{i}}\right]$, so Theorem 4.8 implies that $\underline{i} \subseteq \underline{s} \subseteq \underline{i}$, that is $\underline{i}=\underline{s}$ as desired.

It follows that there are exactly $2^{n}$ classes of the form $\left[B_{\underline{i}}\right]$ because this is the number of possible $\underline{i} \subseteq[n]$.

REMARK 4.10. Assume that $n=3$ and that $\Im_{C_{1}}(R) \subseteq \Im_{C_{2}}(R) \subseteq \Im_{C_{3}}(R)$. The next diagram shows the case of the ordered set of $R$-complexes of the form $B_{\underline{i}}$, with all the reflexivity relations indicated with edges:

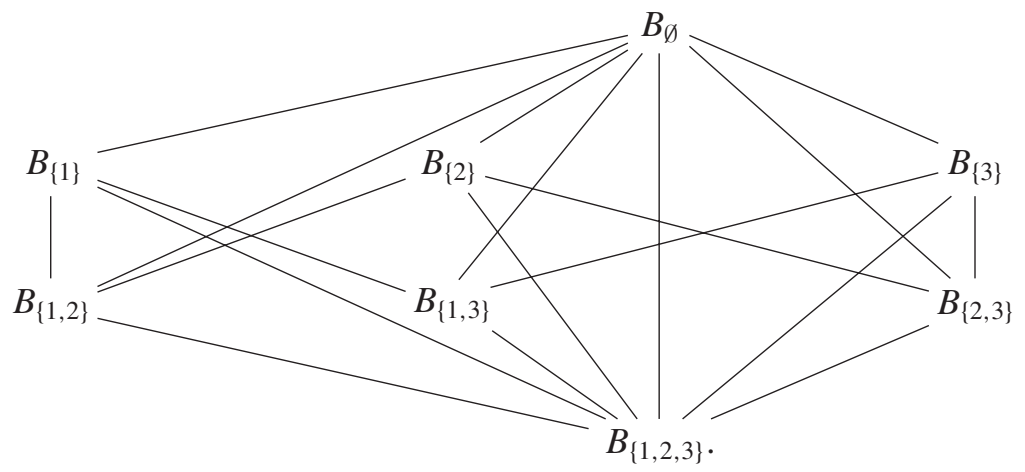

Compare this to the diagram in Remark 3.4.

We conclude with a version of Theorem 4.8 for Auslander classes.

THEOREM 4.11. Assume that $\Xi_{C_{1}}(R) \subseteq \cdots \subseteq \subseteq_{C_{n-1}}(R) \subseteq \subseteq_{C_{n}}(R)$. Under the hypotheses of Assumption 4.1, the following conditions are equivalent:

(i) One has $B_{\underline{\underline{i}}} \in \mathscr{A}_{B_{\underline{\underline{s}}}}(R)$;

(ii) One has $B_{\underline{s}} \in \mathscr{A}_{B_{i}}(R)$;

(iii) The R-complex $B_{\underline{\underline{i}}} \otimes_{R}^{\mathbf{L}} B_{\underline{s}}$ is semidualizing; and

(iv) One has $\underline{i} \cap \underline{s}=\emptyset$.

Proof. The equivalences (i) $\Longleftrightarrow$ (ii) $\Longleftrightarrow$ (iii) are from Remark 2.11.

(iv) $\Longrightarrow$ (iii): If $\underline{i} \cap \underline{s}=\emptyset$, then $B_{\underline{i}} \otimes_{R}^{\mathbf{L}} B_{\underline{s}} \simeq B_{\underline{i} \cup \underline{s}}$, which is semidualizing by Lemma 4.5 .

(iii) $\Longrightarrow$ (iv): Assume that $B_{\underline{i}} \otimes_{R}^{\mathrm{L}} B_{\underline{s}}$ is a semidualizing $R$-complex, and suppose that $a \in \underline{i} \cap \underline{s}$. It follows that we have $B_{\underline{i}} \otimes_{R}^{\mathbf{L}} B_{\underline{s}} \simeq B_{a} \otimes_{R}^{\mathbf{L}} B_{a} \otimes_{R}^{\mathbf{L}} X$ 
where $X=B_{\underline{i} \backslash\{a\}} \otimes_{R}^{\mathbf{L}} B_{\underline{s}} \backslash\{a\}$. Remark 2.8.4 implies that $B_{a} \sim R$, and hence

$$
\begin{aligned}
C_{a-1} & \simeq \mathbf{R H o m}_{R}\left(\mathbf{R H o m}_{R}\left(C_{a-1}, C_{a}\right), C_{a}\right) \\
& \simeq \mathbf{R H o m}_{R}\left(B_{a}, C_{a}\right) \\
& \sim \mathbf{R H o m}_{R}\left(R, C_{a}\right) \\
& \simeq C_{a} .
\end{aligned}
$$

This contradicts the condition $\left[C_{a}\right] \neq\left[C_{a-1}\right]$ which is part of the assumption $\left[C_{a}\right] \triangleleft\left[C_{a-1}\right]$. Thus, we must have $\underline{i} \cap \underline{s}=\emptyset$.

\title{
REFERENCES
}

1. Avramov, L. L., and Foxby, H.-B., Locally Gorenstein homomorphisms, Amer. J. Math. 114 (1992), 1007-1047.

2. Avramov, L. L., and Foxby, H.-B., Ring homomorphisms and finite Gorenstein dimension, Proc. London Math. Soc. (3) 75 (1997), 241-270.

3. Avramov, L. L., Foxby, H.-B., and Lescot, J., Bass series of local ring homomorphisms of finite flat dimension, Trans. Amer. Math. Soc. 335 (1993), 497-523.

4. Christensen, L. W., Semi-dualizing complexes and their Auslander categories, Trans. Amer. Math. Soc. 353 (2001), 1839-1883.

5. Christensen, L. W., and Sather-Wagstaff, S., A Cohen-Macaulay algebra has only finitely many semidualizing modules, Math. Proc. Cambridge Philos. Soc. 145 (2008), 601-603.

6. Frankild, A., and Sather-Wagstaff, S., Reflexivity and ring homomorphisms of finite flat dimension, Comm. Algebra 35 (2007), 461-500.

7. Frankild, A., and Sather-Wagstaff, S., The set of semidualizing complexes is a nontrivial metric space, J. Algebra 308 (2007), 124-143.

8. Frankild, A. J., Sather-Wagstaff, S., and Taylor, A., Relations between semidualizing complexes, J. Commut. Algebra 1 (2009), 393-436.

9. Gelfand, S. I., and Manin, Y. I., Methods of Homological Algebra, Springer, Berlin 1996.

10. Gerko, A. A., On the structure of the set of semidualizing complexes, Illinois J. Math. 48 (2004), 965-976.

11. Hartshorne, R., Residues and Duality, Lecture Notes in Math. 20, Springer, Berlin 1966.

12. Sather-Wagstaff, S., Bass numbers and semidualizing complexes, pp. 349-381 in: Commutative Algebra and its Applications, Proc. Fez 2008, de Gruyter, Berlin 2009.

13. Verdier, J.-L., Catégories dérivées, pp. 262-311 in: Séminaire de Géometrie Algébrique du Bois Marie SGA 4 $\frac{1}{2}$, Lecture Notes in Math. 569, Springer, Berlin 1977.

14. Verdier, J.-L., Des catégories dérivées des catégories abéliennes, Astérisque 239 (1996), $1-253$.

\author{
DEPARTMENT OF MATHEMATICS \\ NDSU DEPT \# 2750 \\ PO BOX 6050 \\ FARGO, ND 58108-6050 \\ USA \\ E-mail: sean.sather-wagstaff@ndsu.edu \\ URL: http://www.ndsu.edu/pubweb/ $\sim$ ssatherw/
}

